\title{
Morphological assessment of sucrose preservation for porcine heart valves
}

\author{
PJ DRURY, EGJ OLSEN, DN ROSS
} From the Department of Surgery, Cardiothoracic Institute, London, and the Department of Pathology,
National Heart Hospital, London

ABSTRACT Porcine aortic valves stored in various concentrations of sucrose $(50-80 \%)$ for up to 52 weeks were examined both histologically and by electron microscopy. The valves were compared with porcine aortic valves stored in a nutrient and antibiotic medium for 12 weeks. Overall preservation was better in those porcine valves stored in sucrose solution than in nutrient and antibiotic medium, the best preservation being in 50\% sucrose. Despite wide separation of collagen at that concentration seen on electron microscopy (not noted histologically), tissue preservation was good after storage in sucrose solutions at concentrations up to $80 \%$, at which clumping of collagen and distortion of fibroblasts occurred. It is suggested that sucrose solution is acceptable for the long-term preservation of biological valves.

Owing to the slow decline in physical properties ${ }^{1}$ and viability, ${ }^{2}$ fresh human valves stored in a nutrient medium at $4^{\circ} \mathrm{C}$ have a limited storage time of eight weeks. The only advantage of a combination of glycerol and nutrient medium is that storage can be maintained at room temperature. ${ }^{3}$ Other attempts to overcome these problems, such as by freeze drying and flash freezing, ${ }^{45}$ have not proved entirely satisfactory.

Sucrose has been used for many years in the food industry as an alternative method of preservation. Through osmotic activity it effectively lowers the moisture available to support microbial growth. ${ }^{6}$ This process, which maintains the ionic balance of the cells and thereby preserves the basic structure of the tissues, can be reversed almost immediately. The use of sucrose as a preserving agent for biological tissues in clinical applications would be a major departure from earlier methods. We have therefore undertaken a morphological examination of biological valves stored in different concentrations of sucrose for up to 52 weeks.

\section{Methods}

VALVE PREPARATION

Porcine aortic valves stored in sucrose solutionsTwelve aortic valves were taken from pig hearts

Address for reprint requests: Dr PJ Drury, Department of Medical Physics and Clinical Engineering, Royal Hallamshire Hospital, Sheffield S10 2JF. delivered to the laboratory within six hours of slaughter. The valves were immediately dissected, immersed in Hartmann's solution at $4^{\circ} \mathrm{C}$, and stored overnight. Sucrose solutions, $50 \%, 60 \%, 70 \%$, and $80 \%$ by weight, were made up, the sucrose being dissolved in distilled water by boiling. The solutions were covered and allowed to cool to room temperature. The valves were removed from the Hartmann's solution and divided longitudinally into three portions, each piece containing a cusp and part of the aortic wall. Each piece was placed in a separate container of the same concentration of sucrose. After all air had been removed, the valve pieces were stored at $4^{\circ} \mathrm{C}$ for three, 12 , and 52 weeks respectively. The process was repeated three times at each concentration of sucrose (there were 36 valve cusps). At the end of the required storage periods the valves were transferred for histological examination. Four valves were similarly treated but stored in sucrose for 24 hours only. A further three valves were transferred directly from the Hartmann's solution for histological examination. These latter seven valves were the controls for this part of the investigation. Thirty-six valves were stored in sucrose for three, 12, and 52 weeks - three valves at each sucrose concentration for each time period. These valves were reconstituted in several $200 \mathrm{ml}$ washes of Hartmann's solution, which was changed at two-minute intervals for 10 minutes and thereafter at 10-minute intervals. The times required for complete reconstitution and the macroscopic changes in the valves were noted.

Porcine aortic valves stored in nutrient and antibio- 
tic medium-Three pig aortic valves were dissected, washed, and stored overnight in Hartmann's solution as before. The valves were transferred to a nutrient and antibiotic medium ${ }^{7}$ for 12 weeks, after which time they were processed for histological examination. This time period was chosen because it has been shown previously ${ }^{12}$ that valves preserved in such a medium have a limited storage time of eight weeks.

\section{HISTOLOGICAL PREPARATIONS}

Samples of the valves were removed from the sucrose solutions or nutrient-antibiotic r redium, washed five times, and fixed in $10 \%$ buffered neutral formalin. Subsequently representative blocks were selected from each piece of tissue to include a continuum of valve leaflet and aortic wall. The tissue was then processed for paraffin embedding and $5-\mu \mathrm{m}$-thick sections were cut and stained by haematoxylin and eosin and Miller's elastic and Van Gieson. Particular attention was paid to the architecture of the valve leaflets, their thickness, and the integrity of the collagen tissue.

To provide some idea of the changes observed histologically, a semiquantitative approach using a plus system was used.

Assessment of thickening due to oedema-++ confluent oedema resulting in thickening of the valve leaflet; + one-half of the valve leaflet affected; \pm one-third of the valve leaflet affected; \pm focal distribution. The thickening, when present, was assessed in the middle third of the valve leaflet (base to tip) and measured with an eyepiece graticule.

Necrosis of the collagen component of the valve leaflet invariably focal -++ at least one focus, high-power field (magnification $\times 250$ ); + at least one focus per two fields; \pm at least one focus per three fields; \pm no more than two foci in the entire biopsy specimen.

\section{ELECTRON MICROSCOPY PREPARATION}

Pieces of the valve leaflets from the preserved valves were selected and washed several times and fixed in $3 \%$ glutaraldehyde in $0.2 \mathrm{~mol} / \mathrm{l}$ sodium cacodylate for one hour at $4^{\circ} \mathrm{C}$. The solution was then changed to sodium cacodylate-sucrose washing solution for one hour at $4^{\circ} \mathrm{C}$. The tissues were post fixed in $1 \%$ osmium tetroxide in $\mathrm{PO}_{4}$ buffer for one hour at $4^{\circ} \mathrm{C}$ and then processed and flat-embedded in Epon 812 mixture by standard procedures.

Sections $50 \mathrm{~nm}$ thick were cut on a Porter-Blum MTI microtome. The sections, mounted on 300mesh copper grids, were stained by uranyl acetate and lead citrate. Viewing was carried out with a Philips 301 electron microscope.

\section{Results}

\section{STORAGE IN SUCROSE SOLUTIONS}

Macroscopy-Porcine aortic valves were removed from the sucrose solutions at three, 12, and 52 weeks, reconstituted in Hartmann's solution, and inspected macroscopically for signs of deterioration in appearance, texture, or odour. After three weeks' storage the valves could be reconstituted within five minutes of their removal from the sucrose and appeared identical to the fresh, control valves except for a slight brownish discolouration of the muscular parts of the valve. After 12 weeks' storage the valves were reconstituted within eight to 12 minutes of removal from the sucrose. The valves again appeared no different from the control valves except that the muscular parts were definitely discoloured. After 52 weeks' storage only the aortic cusps of the valves could be reconstituted within 10 minutes and again were identical in appearance and texture to the control cusps. The aortic wall, however, remained leathery and of a greyish appearance.

Histology-The normal architecture was preserved in all samples examined and the histological findings are summarised in the table. Normal valve leaflets were examined as controls and an example is given in figure 1 . No change from the normal control was noted after 24 hours' storage in any of the sucrose solutions used. After three weeks in $50 \%$ sucrose normal integrity of the tissues was maintained. One sample from each of the valve leaflets examined after storage in both $60 \%$ and $80 \%$ sucrose showed mild swelling due to fluid accumulations. Apart from

Morphological changes in porcine aortic valves stored in sucrose solution

\begin{tabular}{|c|c|c|c|c|c|}
\hline Sucrose \% & $\begin{array}{l}\text { Morphological } \\
\text { change* }\end{array}$ & $24 h$ & $3 w$ & $12 w$ & $52 w$ \\
\hline \multirow[b]{2}{*}{50} & Oedema & - & - & - & - \\
\hline & Necrosis & - & - & - & - \\
\hline \multirow{3}{*}{60} & Oedema & - & \pm & \pm & \pm \\
\hline & Necrosis & - & - & \pm & \pm \\
\hline & Oedema & - & \pm & + & \pm \\
\hline 70 & Necrosis & - & $=$ & \pm & \pm \\
\hline \multirow{2}{*}{80} & Oedema & - & \pm & \pm & + \\
\hline & Necrosis & - & - & + & \pm \\
\hline \multirow{2}{*}{$\begin{array}{l}\text { Nutrient-antibiotic } \\
\text { medium: }\end{array}$} & Oedema & $x$ & $x$ & ++ & $\times$ \\
\hline & Necrosis & $\times$ & $\times$ & + & $\times$ \\
\hline
\end{tabular}

*Oedema: ++ confluent oedema resulting in thickening of the valve leaflet; + involvement of one-half of the valve leaflet; \pm involvement of one-third of the valve leaflet; \pm focal distribution; - no oedema present; $x$ not assessed. Necrosis: ++ at least one focus, high-power field (magnification $\times 250$ ); + at least one focus per two fields; \pm at least one focus per three fields; \pm no more than two foci in the entire biopsy specimen; - no necrosis present; $x$ not assessed. The symbols indicate the worst changes observed in each group of three valves in each sucrose concentration and duration category. 


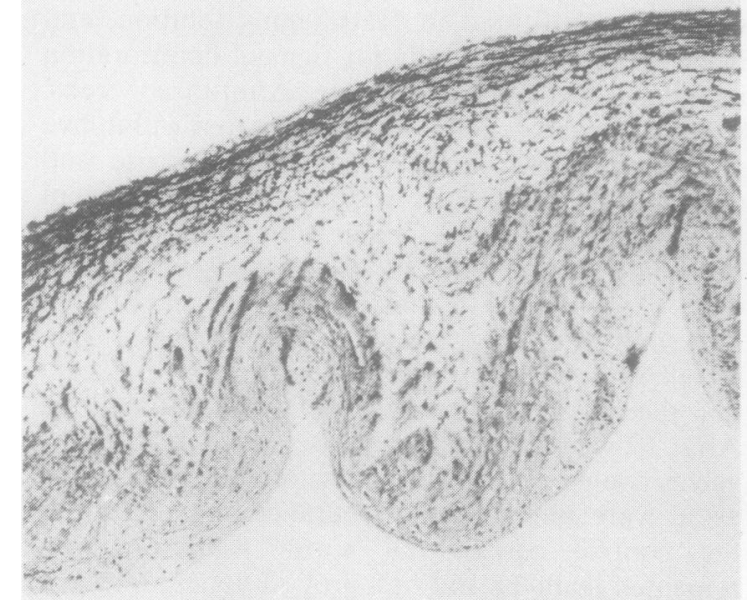

Fig 1 Normal valve leaflet showing the three layers. The elastic layer (top of the photomicrograph) is separated from the lower fibrous layer by the zona spongiosa composed of loose connective tissue. Miller's elastic and Van Gieson, $\times$ 80.

occasional small foci of accumulated fluid, in valves stored in higher concentrations of sucrose for 12 weeks the architectural integrity was normal. Good preservation was maintained in $50 \%$ sucrose after 52 weeks (fig 2), but with $60 \%$ solutions focal necrosis of the collagen was observed (fig 3). In 70\% and $80 \%$ sucrose, however, no significant changes were observed. The integrity of the aortic wall was well preserved in all sucrose concentrations over all the time periods used.

Electron microscopy-Samples of tissue were examined after storage in the sucrose solutions for 24 hours and 52 weeks. The normal structure included bundles of collagen arranged in a wavy pattern. Occasional foci of irregularity were noted. Varying degrees of prominence of the matrix were also observed (fig 4). After 24 hours there was no significant change from the control apart from some separation of collagen bundles by amorphous material. This separation was interpreted as due to fluid

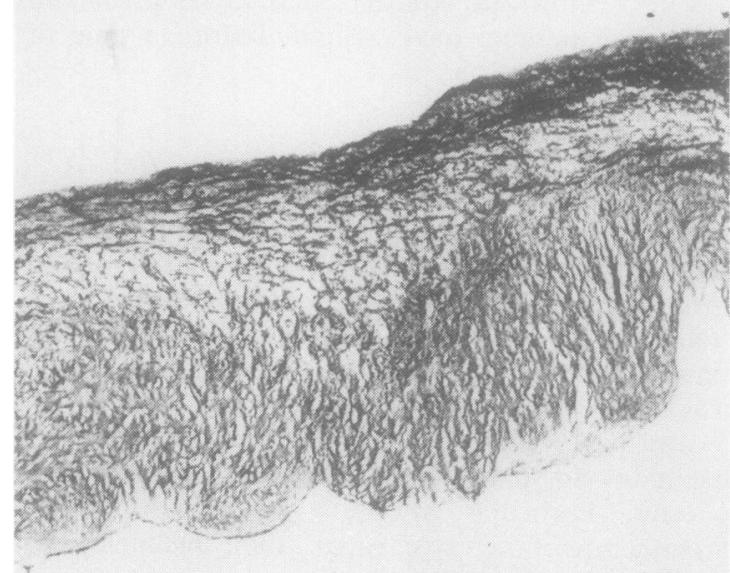

Fig 2 Part of the valve leaflet after storage for 52 weeks in $50 \%$ sucrose, showing normal preservation of the three layers and normal integrity of the collagen in the fibrous layer. Miller's elastic and Van Gieson $\times 80$.

accumulation. After 52 weeks' storage in $50 \%$ and $60 \%$ sucrose, collagen fibrils were widely separated by such fluid accumulations in places, but the collagen fibrils themselves showed normal periodicity. Fibroblasts were fewer in number. This focal widening was more prominent in tissue stored in $60 \%$ sucrose than that stored in $50 \%$ sucrose. The severest changes were observed after storage in $80 \%$ sucrose. Clumping of the collagen had occurred at this concentration but there was maintenance of periodicity. Accumulations of fluid were widespread and the fibroblasts were extensively distorted (fig 5).

STORAGE IN NUTRIENT AND ANTBIOTIC MEDIUM The aortic valves were sampled at 12 weeks and compared histologically with those stored in the sucrose solutions for a similar period. Variable focal degeneration of collagen was noted with moderateto-severe accumulation of fluid. From the histological examination we concluded that the tissue was 


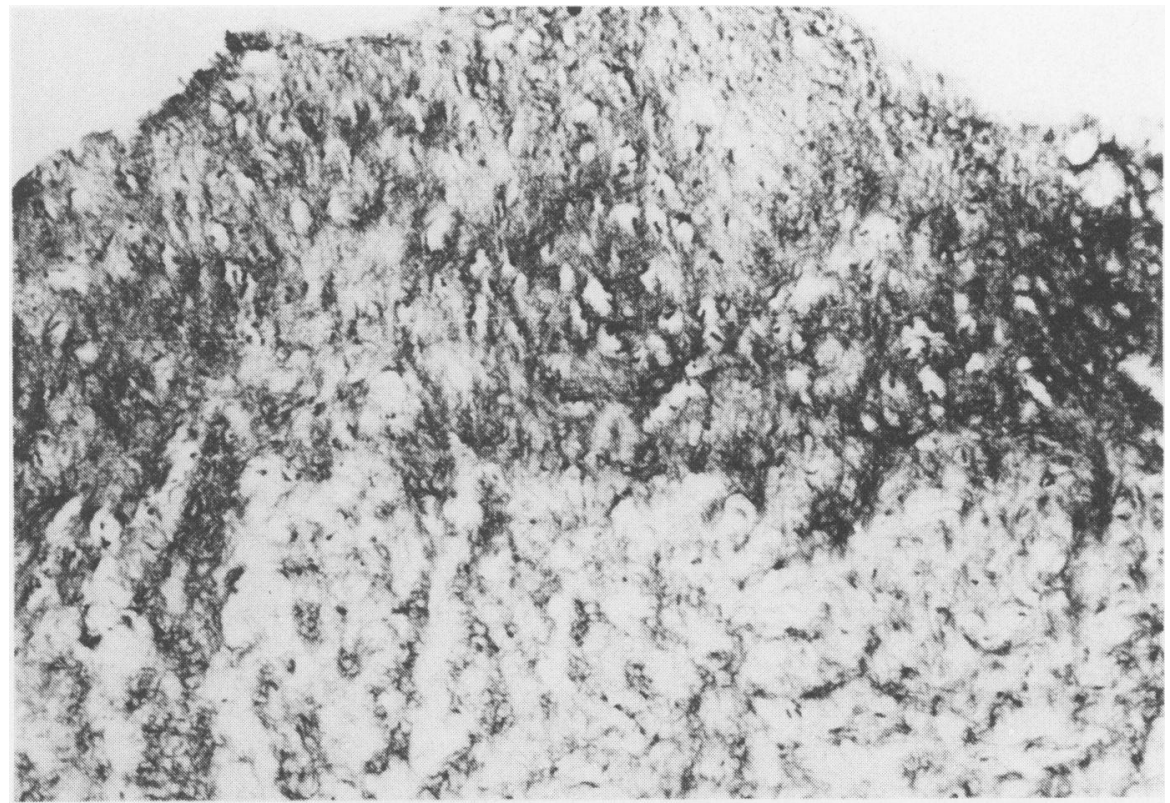

Fig 3 Close-up photomicrograph of an area of collagen tissue in the fibrous layer after storage of this valve leaflet in $60 \%$ sucrose for 52 weeks. Note the focal areas of necrosis (loss of fibrillar arrangement). Miller's elastic and Van Gieson $\times 200$.

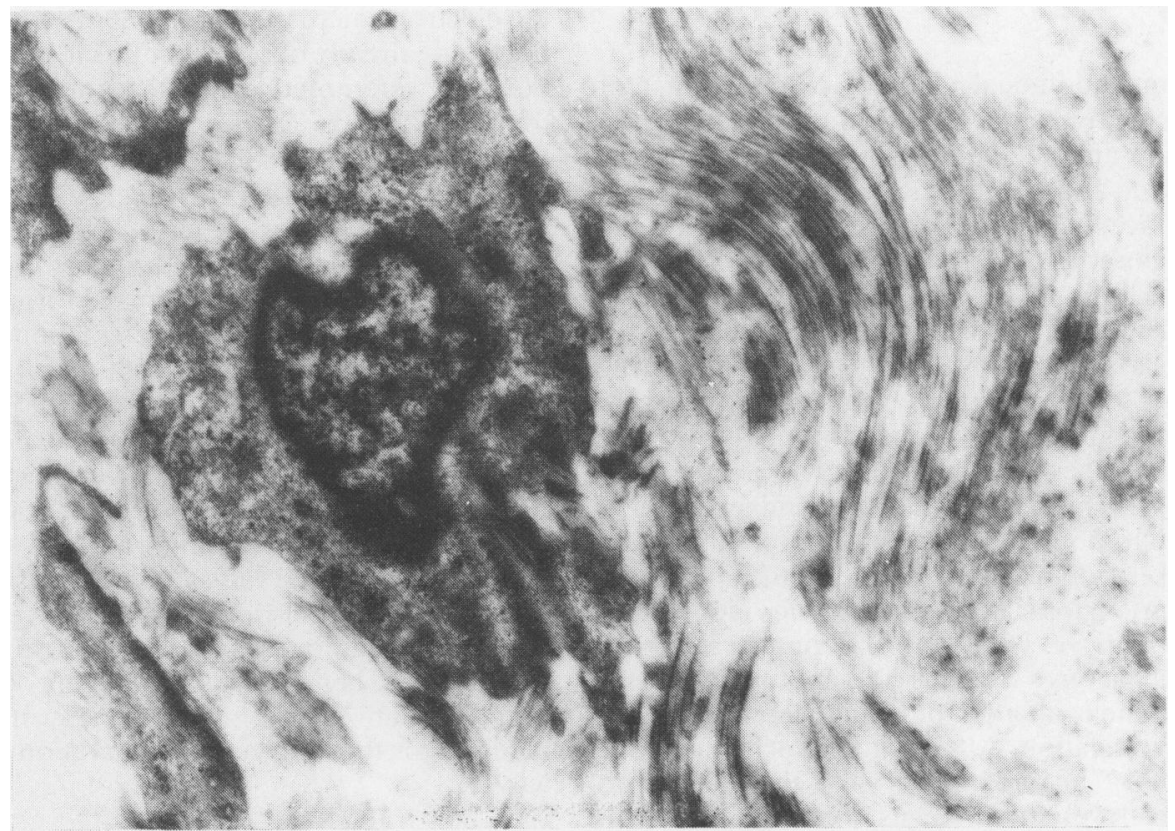

Fig 4 Electron micrograph of part of the normal valve leaflet showing the arrangement of the collagen. Uranyl acetate and lead citrate $\times 13125$. 


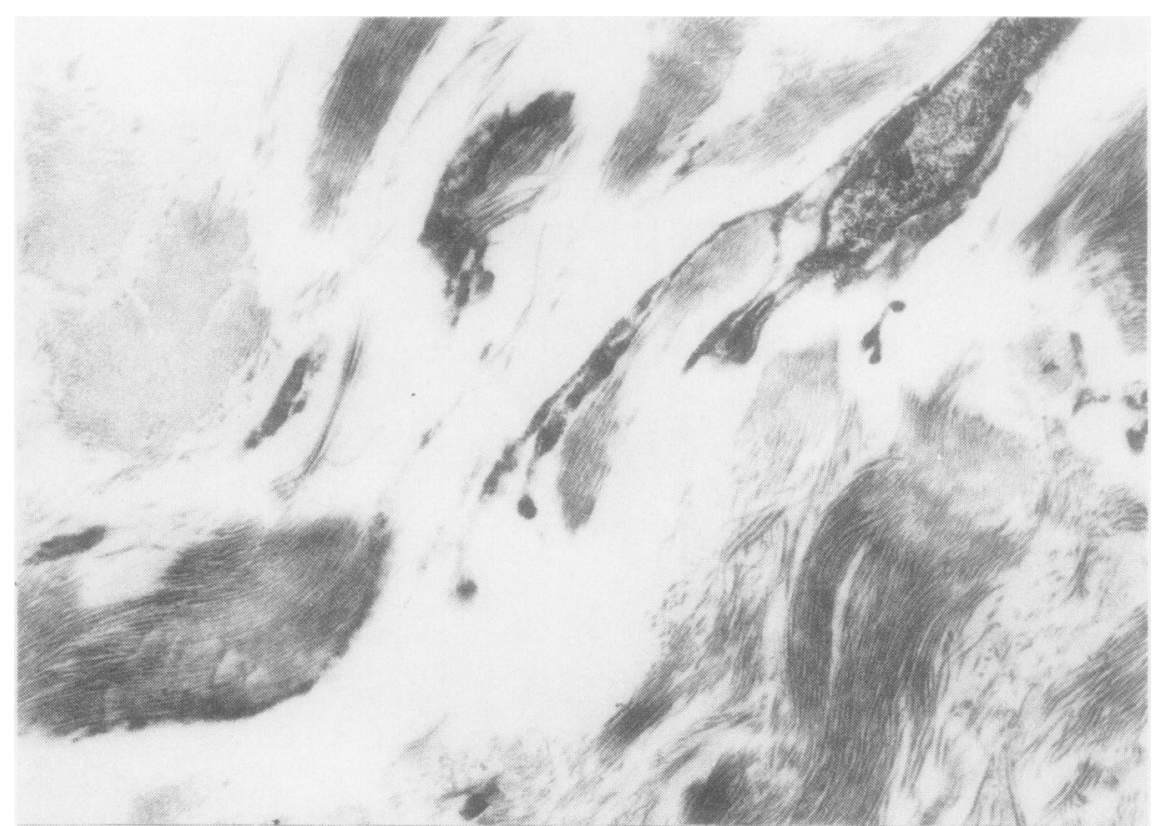

Fig 5 Electron micrograph of part of the valve leaflet after storage for 52 weeks in $80 \%$ sucrose showing fluid accumulations and distorted fibroblast. Uranyl acetate and lead citrate $\times 11180$.

better preserved in sucrose than in the nutrient and antibiotic medium.

\section{Discussion}

Apart from endothelial cells all valves consist of three basic components. Collagen and elastic tissue are present; and the fibrous layer, known as the holding face, and the elastic layer, known as the deformed face, ${ }^{8}$ are separated by the third layer, the zona spongiosa, a loose connective tissue of varying prominence. ${ }^{9}$

Minor degrees of thickening of valve leaflets are difficult to assess because slight angulation during preparation for histological examination may give an erroneous impression of the normal valve thickness. Only significant thickening can be assessed and none was present in the material examined.

Wide separation of collagen by amorphous material was observed and interpreted as fluid. Although the zona spongiosa is known to vary in prominence, ${ }^{10}$ when we were interpreting the electron microscopic pictures we took care not to include this structure in the evaluation of the appearances at that level of investigation. Although fluid accumulations were noted histologically, at the electronmicroscopic level the changes were more severe. This is not altogether surprising as examination is carried out at much higher magnification and there- fore the earliest changes would first be observed at that level of investigation. Histologically $50 \%$ sucrose solutions afforded the best preservation, but at the electron-microscopic level fluid and wide separation were already observed at this lowest concentration and were noted least after storage in $80 \%$ sucrose for 52 weeks. At this latter concentration, however, clumping of collagen was evident and was interpreted as the severest change. The periodicity of the collagen was nevertheless preserved. The storage of tissues in supersaturated solutions of sucrose might result in sucrose crystal formation, which caused separation of the tissue components. During the washing phase of the histological preparation these crystals would dissolve and be replaced by water through osmosis. This would explain the fluid accumulations, which were especially noted after storage in the higher sucrose concentrations.

Although we noted minor changes in various components of the valves stored in sucrose solutions, after comparison with those valves stored in a nutrient and antibiotic medium sucrose solution was seen to be a better storage medium. We therefore suggest that sucrose solution is an acceptable medium for the long-term preservation of biological valves.

The authors gratefully acknowledge the help of Haverhill Meat Products and J Sainsbury for their 
generous supplies of porcine material. The authors would also like to thank Carol Outram and Carol Cheetham for their help in preparing the manuscript.

\section{References}

' $\mathrm{Ng}$ YL, Wright JEC. Effect of preservation on the elasticity of human aortic valve homografts. Thorax 1975;30:266-70.

${ }^{2}$ Al-Janabi N, Ross DN. Enhanced viability of fresh aortic homografts stored in nutrient medium. Cardiovasc Res 1973;7:817-22.

${ }^{3}$ Parker R, Randev R, Wain WH, Ross DN. Storage of heart valve allografts in glycerol with subsequent antibiotic sterilisation. Thorax 1978;33:638-45.
${ }^{4}$ Longmore DB, Lockey E, Ross DN, Pickering BN. The preparation of aortic valve homografts. Lancet 1966;ii:463-4.

${ }^{5}$ Moore CH, Martelli V, Al-Janabi N, Ross DN. Analysis of homograft valve failure in $\mathbf{3 1 1}$ patients followed up to ten years. Ann Thorac Surg 1975;20:274-81.

- Brooke M. In: Yudkin J, Edelman J, Hough L, eds. Sugar. London: Butterworths, 1971.

${ }^{7}$ Wain WH, Pearce HM, Riddell RW, Ross DN. A re-evaluation of antibiotic sterilisation of heart valve allografts. Thorax 1977;32:740-2.

B Olsen EGJ. The pathology of the heart. 2nd ed. London: Macmillan, 1980:22.

- Gross L, Kugel MA. Topographic anatomy and histology of the valves in the human heart. Am J Path 1931;7:445-74.

${ }^{10}$ Olsen EGJ, Al-Rufaie HK. The floppy mitral valve. Study on pathogenesis. Br Heart J 1980;44:674-83. 